\title{
Efficacy and safety of oral recombinant calcitonin tablets in postmenopausal women with low bone mass and increased fracture risk: a randomized, placebo-controlled trial
}

\author{
N. Binkley • H. Bone • J. P. Gilligan • D. S. Krause
}

Received: 12 June 2014 / Accepted: 25 June 2014 / Published online: 16 July 2014

(C) The Author(s) 2014. This article is published with open access at Springerlink.com

\begin{abstract}
Summary The effect of an investigational oral calcitonin tablet upon bone mineral density (BMD) of the spine was investigated in postmenopausal women with low bone mass and at increased risk of fracture. Compared to placebo, calcitonin tablets increased lumbar spine BMD. This agent may provide an additional choice for patients.

Introduction An investigational oral salmon calcitonin preparation was previously shown to increase lumbar spine BMD in postmenopausal women with osteoporosis. Our objective was to evaluate the use of this agent in postmenopausal women with low bone mass and at increased fracture risk but not meeting BMD criteria for osteoporosis.

Methods Treatment-naïve women were randomized to receive oral recombinant salmon calcitonin tablets or placebo once daily for 1 year. Dual-energy X-ray absorptiometry was performed at baseline and at study weeks 28 and 54. CTx-1, a bone resorption marker, was obtained at the same time points. Subjects returned periodically for tolerability assessment and adverse event (AE) recording.

Results One hundred twenty-nine women in the USA were randomized, 86 to calcitonin and 43 to placebo. Calcitonin recipients experienced a significant increase from baseline in lumbar spine BMD; the difference compared with placebo was significant. Dosing at bedtime or with dinner was equally effective. CTx-1 was suppressed in calcitonin recipients but
\end{abstract}

N. Binkley $(\bowtie)$

University of Wisconsin Osteoporosis Clinical Center and Research

Program, Madison, WI, USA

e-mail: nbinkley@wisc.edu

H. Bone

Michigan Bone and Mineral Clinic, Detroit, MI, USA

J. P. Gilligan • D. S. Krause

Tarsa Therapeutics Inc., Philadelphia, PA, USA not in placebo subjects. Gastrointestinal AEs were common, but the overall safety profile was comparable between groups. Conclusions Oral calcitonin may provide a useful therapeutic alternative for some women with low bone mass.

Keywords Bone mineral density · Calcitonin · Oral · Osteopenia $\cdot$ Osteoporosis

\section{Introduction}

Osteoporosis occurs most commonly in women, due in part to lower peak bone mass and rapid perimenopausal bone loss [1]. Osteoporosis-related fractures result in substantial morbidity, health care cost, loss of independence, and increased mortality [2]. The majority of these fractures occur in women who do not have osteoporosis as defined by their bone mineral density (BMD) [3]. As a result, much recent effort has focused on improving identification of those who will fracture including use of risk calculators such as the World Health Organization's FRAX ${ }^{\circledR}$ tool [4]. Indeed, it has recently been suggested that osteoporosis be diagnosed in those at increased fracture risk $[5,6]$.

In spite of these personal and societal costs, and in the face of an aging population, use of medications to reduce fracture risk has declined dramatically, even following hip fracture [7, $8]$. This decline is certainly multifactorial but is likely due in large part to concerns about side effects including rare complications such as osteonecrosis of the jaw (ONJ) and atypical femoral fractures (AFFs). As such, a clear need exists for medications that safely reduce bone loss with advancing age.

The calcitonins are 32 amino acids, naturally occurring calcium-regulating peptide hormones produced by the thyroid glands in mammals and the ultimobranchial glands in birds and fish [9]. Calcitonins have long been used as therapeutic agents because of their antiresorptive properties, effected by 
intracellular second messengers following binding to specific $\mathrm{G}$ protein-coupled receptors on osteoclasts, resulting in nonapoptotic inhibition of osteoclastic activity [10]. Pharmaceutical preparations in Europe and the USA generally employ synthetic salmon calcitonin, which is regarded as a more potent antiresorptive than human calcitonin [11]. In the USA, recombinant and synthetic salmon calcitonin nasal sprays and synthetic injectable formulations are approved for osteoporosis treatment in women who are at least 5 years postmenopause and for whom other agents are contraindicated or refused [12].

Nasal calcitonin is well tolerated but produces only modest blood levels; an oral preparation might provide a desirable alternative for some patients and might help to improve initial acceptance of therapy and subsequent compliance. However, oral delivery of peptide agents is challenging, principally due to enzymatic digestion in the proximal gastrointestinal tract. We have previously demonstrated that an investigational oral recombinant salmon calcitonin preparation is bioavailable and increases lumbar spine BMD to a greater degree than calcitonin nasal spray in postmenopausal women with established osteoporosis [13]. Because bone resorption is greatest overnight and the effect of calcitonin is transient, the investigational calcitonin preparation was administered in the evening [14]. In the present study, we evaluated this preparation in postmenopausal women with osteopenia and an elevated fracture risk as calculated using the FRAX tool, who did not meet a T-score definition of osteoporosis [15]. The entry criteria for elevated risk in this study were designed to mirror the US Preventative Services Task Force recommendations for identifying women with increased risk of osteoporosis [16]. We also explored administration with the evening meal compared to bedtime dosing.

\section{Materials and methods}

\section{Participants}

Women $\geq 45$ years of age and at least 5 years postmenopause with BMD T-score $<-1.0$ but $>-2.5$ at the lumbar spine, total hip, femoral neck, or trochanter were eligible for the study. Eligible subjects had serum FSH $\geq 0 \mathrm{mIU} / \mathrm{mL}$, serum 25 hydroxyvitamin $\mathrm{D} \geq 20 \mathrm{ng} / \mathrm{mL}$, and body mass index (BMI) $\leq 35 \mathrm{~kg} / \mathrm{m}^{2}$. Subjects were also required to have clinical risk factors such that the 10-year risk of major osteoporotic fracture as determined by the FRAX algorithm was at least as great as a 65-year-old woman of the same race with a BMI of $25 \mathrm{~kg} / \mathrm{m}^{2}$, and without additional risk factors. As an example, for a 65-year-old (US) white woman with this BMI, the 10year risk levels of a major osteoporotic fracture or hip fracture are 9.3 and $1.2 \%$, respectively. Major exclusion criteria were history of osteoporotic fracture, T-score $\leq-2.5$ at any of the sites listed above, treatment with hormone replacement therapy within 3 months of randomization, and prior use of calcitonin, bisphosphonates, denosumab, fluoride, strontium, or parathyroid hormone analogs.

\section{Study design}

Two screening visits occurred after written informed consent was obtained. At the first screening visit (visit 0 ), the 10-year risk of a major osteoporosis fracture or hip fracture was assessed using the WHO FRAX tool ("FRAX1", i.e., without BMD data). At the next screening visit (visit 1), within 30 days of visit 0 , a BMD determination of the anterior-posterior lumbar spine, femoral neck, trochanter, and total hip was performed via dual energy x-ray absorptiometry (DXA). In addition, a baseline blood sample was drawn for determination of C-terminal telopeptide of type 1 collagen (CTx-1), a biochemical marker of bone resorption.

At or following visit 2, which occurred within 30 days of the first screening visit (visit 0 ), the FRAX risk was recalculated including the femoral neck BMD value obtained at visit 1 ("FRAX2"). If the subject met all study inclusion criteria (either FRAX calculation could serve as the qualifying criterion), she could be enrolled. Eligible subjects were then given a 14-day supply of placebo study medication and instructed to take this at either bedtime or dinner time for a 2-week single-blind (i.e., subject blinded) run-in phase. Subjects were also provided $600 \mathrm{mg}$ calcium citrate and 1,000 IU vitamin D supplements to take once daily with breakfast throughout the study. The purposes of the run-in phase were to determine tolerability of the excipients used in the formulation of both the placebo and oral calcitonin and also of the calcium citrate and vitamin D supplementation and to confirm subject compliance.

At visit 3, 2 weeks after beginning the run-in period, each subject's eligibility for randomization to double-blind treatment was determined based on all prior screening results, tolerability of the blinded placebo study medication during the run-in phase, and compliance with the assigned placebo medication and supplement. If a subject was deemed ineligible due to lack of tolerability, refused to continue, or was not compliant, any treatment-emergent adverse events (TEAEs) were documented, and the subject was discontinued from the study. Eligible, continuing subjects were randomized in a 2:1 ratio to receive identical appearing oral calcitonin or placebo tablets. Initially, subjects were to take the assigned medication at bedtime on an empty stomach. Following receipt of data from a phase 1 study that suggested no food effect on the pharmacodynamic response (i.e., suppression of serum CTx-1 levels) to the oral calcitonin preparation, the protocol was modified during the study's enrollment period so that newly enrolling subjects were instructed to take oral calcitonin or placebo with the evening meal. 
The study duration was 54 weeks, including the 2-week run-in phase. BMD and CTx-1 were measured at the second screening visit (visit 1), week 28 (visit 6), and week 54 (visit 8). Subjects experiencing a BMD loss of $\geq 7 \%$ at any measured site at week 28 were to be discontinued from the study.

\section{Treatments}

Study volunteers were provided identical appearing tablets containing either $0.2 \mathrm{mg}(200 \mu \mathrm{g} / 1,200 \mathrm{IU})$ of recombinant (Escherichia coli) salmon calcitonin or placebo. The formulation of this product has previously been reported [13]. Briefly, each tablet core contains $0.2 \mathrm{mg}$ of recombinant salmon calcitonin and $500 \mathrm{mg}$ of citric acid (CA). The tablet cores have an acid-resistant enteric coat, which dissolves in the basic environment of the proximal small bowel, releasing the $\mathrm{CA}$ and calcitonin. The $\mathrm{CA}$ provides a lower $\mathrm{pH}$ acidic microenvironment, inhibiting endogenous enzymes in the proximal small bowel, which function optimally in a basic medium. Oral calcitonin and placebo were supplied by the sponsor. Calcium and vitamin D supplements were obtained commercially by each clinic site.

Participants self-administered the assigned tablet daily with a glass of water approximately $1 \mathrm{~h}$ before sleeping, until the protocol amendment changed the time of study drug administration to be with the evening meal.

\section{Bone density measurement}

BMD was measured at the lumbar spine (L1-L4) and proximal femur (the neck, trochanter, and total hip) using replicate DXA scans at screening, week 28 and the week 54 final visit. BMD results were averaged if the two scans were within $5 \%$ of each other. If the results fell outside of this range, a third scan was obtained, and the two scans closest to one another were averaged. Each participant was scanned on the same densitometer throughout the study. All DXA scans were reviewed independently in blinded fashion by two expert technicians at a central imaging facility (BioClinica, Inc., Newtown, PA) for technical adequacy of acquisition and then analyzed in accordance with prespecified standard guidelines.

\section{Bone biomarker}

Fasting blood samples were obtained prior to $11 \mathrm{AM}$ at baseline, week 28, and week 54 for determination of CTx-1, which was measured by Pacific Biomarkers, Inc., Seattle, WA, using a commercial assay (Elecsys ${ }^{\circledR}$, Roche Diagnostics). The precision values and limit of quantitation obtained in validation assays were consistent with the manufacturer's data in the product's label.
Safety assessments

A complete physical examination was performed, and specimens for safety laboratory analyses (clinical chemistry, hematology, and urinalysis) were collected at screening (visit 0). A symptom-directed exam and assessment of adverse events were performed at each subsequent visit, and safety laboratory studies were to be repeated at weeks 28 and 54 or the exit (last) visit.

Statistical analysis

The primary objective was to compare the effects of oral calcitonin to placebo with respect to the change from baseline in lumbar spine BMD measurements over a 54week period (i.e., 52 weeks of treatment). The primary efficacy variable was the percent change from baseline in lumbar spine BMD values over 52 weeks. A modified intent-to-treat (MITT) subject population was used for all efficacy analyses. This population was defined as all subjects who received treatment and had at least one postbaseline BMD value. In subjects who completed week 28 but did not complete the full 54-week period, the data were imputed using the last observation carried forward (LOCF) method. All statistical methods were described in a statistical analysis plan completed prior to any unblinding. To test robustness of the results for the primary efficacy variable, which was based on the mixed effect model repeated measure (MMRM) model with LOCF, a sensitivity analysis was planned, using this MMRM model without LOCF. Secondary efficacy variables included lumbar spine (LS) BMD, as assessed by the percent change between baseline and week 28 and BMD changes at the femoral neck, trochanter, and total hip, as assessed by the percent change between baseline and both weeks 28 and 54 .

Safety and tolerability were assessed through the recording of adverse events (AEs) throughout the study, as well as by review of the safety laboratory determinations performed periodically. The safety population was used for all safety analyses, defined as all subjects who received one dose of study drug after randomization, i.e., following the run-in period.

The sample size was estimated assuming a treatment group difference of $1.5 \%$, a standard deviation of $2.5 \%$, two-sided significance level of 0.05 , power equal to $80 \%$, and a 2:1 randomization. Based on these assumptions, it was determined that a total of 102 subjects would need to be randomized, 68 to the oral calctionin group and 34 to the placebo group. In order to obtain 102 women completing therapy, 120 eligible women meeting the inclusion and exclusion criteria and providing informed consent were to be randomized 2:1 (active/placebo). 
Table 1 Baseline subject characteristics (mean \pm SD)

\begin{tabular}{|c|c|c|c|}
\hline \multirow[t]{2}{*}{ Baseline characteristics } & \multicolumn{3}{|c|}{ Safety population } \\
\hline & $\begin{array}{l}\text { Oral calcitonin } \\
(n=86)\end{array}$ & $\begin{array}{l}\text { Placebo } \\
(n=43)\end{array}$ & $\begin{array}{l}\text { Total }^{\mathrm{a}} \\
(n=129)\end{array}$ \\
\hline Age (years) & $67.5 \pm 6.9$ & $66.6 \pm 5.2$ & $67.2 \pm 6.4$ \\
\hline Race (\% white) & 94.2 & 95.3 & 94.6 \\
\hline Height $(\mathrm{cm})$ & $161.2 \pm 6.5$ & $161.0 \pm 7.0$ & $161.1 \pm 6.6$ \\
\hline BMI $\left(\mathrm{kg} / \mathrm{m}^{2}\right)$ & $25.8 \pm 3.8$ & $26.8 \pm 6.0$ & $26.1 \pm 4.6$ \\
\hline FRAX-2 (\%, major $)^{\mathrm{b}}$ & $11.3 \pm 4.4$ & $11.3 \pm 4.5$ & $11.3 \pm 4.4$ \\
\hline FRAX-2 $(\%, \text { hip })^{b}$ & $2.0 \pm 1.9$ & $2.20 \pm 2.903$ & $2.0 \pm 2.3$ \\
\hline $\begin{array}{l}\text { 25-hydroxyvitamin D } \\
\text { (ng/mL) }\end{array}$ & $35.3 \pm 11.1$ & $39.3 \pm 13.2$ & $36.6 \pm 12.0$ \\
\hline $\begin{array}{l}\text { Lumbar spine } \\
\text { (L1-L4) T-score }\end{array}$ & $-1.15 \pm 0.9$ & $-1.12 \pm 0.9$ & $-1.14 \pm 0.9$ \\
\hline Femoral neck T-score & $-1.69 \pm 0.5$ & $-1.73 \pm 0.3$ & $-1.70 \pm 0.4$ \\
\hline Total hip T-score & $-1.23 \pm 0.5$ & $-1.20 \pm 0.5$ & $-1.22 \pm 0.5$ \\
\hline Trochanter T-score & $-0.95 \pm 0.7$ & $-0.99 \pm 0.6$ & $-0.98 \pm 0.6$ \\
\hline
\end{tabular}

${ }^{\mathrm{a}} p>0.05$ for all pairwise comparisons

${ }^{\mathrm{b}}$ FRAX calculated using femoral neck BMD

\section{Results}

\section{Participant characteristics}

The study was conducted at 11 sites in the USA. Two hundred and eighty volunteers provided informed consent and were screened for entry; of these, 144 were eligible and entered the run-in phase, and 129 were randomized to calcitonin or placebo.

Demographic characteristics of the safety population are displayed in Table 1. Study participants were predominantly white, normal to overweight, vitamin D replete, postmenopausal women with a mean age of 67 years. FRAX scores were slightly lower when calculated with inclusion of femoral neck BMD (FRAX 2) compared to screening (FRAX 1, not shown).
T-scores were lowest at the femoral neck. There were no statistically significant differences between treatment groups for any baseline characteristic.

Participant disposition is presented in Fig. 1. Of 144 women entering the single-blind run-in phase, 129 were randomized. The most common reason for not continuing to randomization was not tolerating the placebo tablet or calcium/ vitamin D supplement ( $N=10$, see "Safety and tolerability" below). Other reasons for failure to proceed to randomization $(N=5)$ included poor compliance or the subsequent identification of exclusion criteria (such as a prior history of fracture or inability to obtain an adequate DXA scan). The 129 subjects proceeding to randomization constitute the safety population. The efficacy analysis was conducted in the MITT population of 114 subjects, 78 in the calcitonin group and 36 in the placebo group. Ninety-nine women completed all study visits. The proportion discontinuing was slightly higher in the placebo group, although the proportion of subjects discontinuing for tolerability reasons was similar between groups (see "Safety and tolerability"). Additional reasons for discontinuation included withdrawal at the subject's request (five placebo, six calcitonin), lost to follow-up (one each), investigator decision, and BMD loss $\geq 7 \%$, one each in the placebo arm.

\section{Bone mineral density}

Changes from baseline in BMD at the various anatomic sites over the course of the study are displayed in Table 2; the change in LS BMD over time is displayed in Fig. 2. Over 54 weeks, calcitonin recipients experienced a significant improvement from baseline in $\operatorname{LS} \operatorname{BMD}(1.03 \%, p<0.001)$, while placebo recipients experienced a nonsignificant decrease in LS BMD from baseline. The difference between groups, $1.14 \%$, was statistically significant $(p=0.027)$. The sensitivity analysis, performed without using the LOCF, also

Fig. 1 Participant disposition

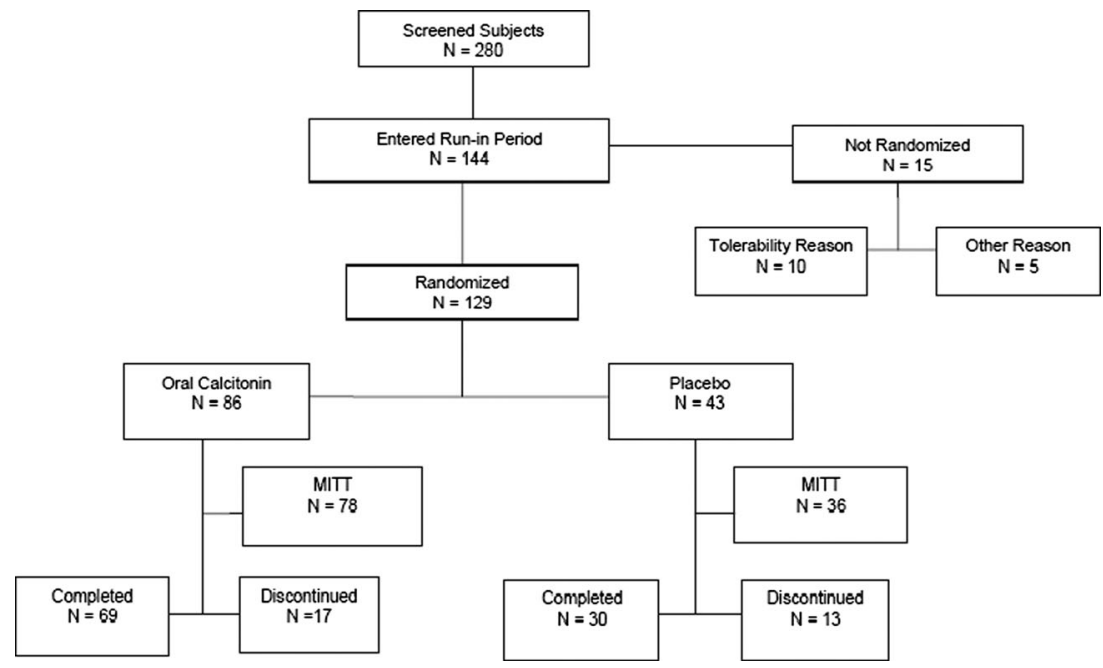


Table 2 Bone mineral density and CTx-1
Data presented as least square mean (95\% confidence intervals)

$p$ Values denote oral calcitonin versus placebo

Bold values differ from baseline $(p<0.05)$ based on within group $t$ test comparison

\begin{tabular}{|c|c|c|c|}
\hline Change from baseline & $\begin{array}{l}\text { Oral calcitonin } \\
N=78\end{array}$ & $\begin{array}{l}\text { Placebo } \\
\mathrm{N}=36\end{array}$ & $p$ Value \\
\hline \multicolumn{4}{|l|}{ Lumbar spine BMD, \% } \\
\hline Week 28 & $0.96(0.38,1.54)$ & $0(-0.84,0.85)$ & 0.06 \\
\hline Week 54 & $1.03(0.46,1.59)$ & $-0.12(-0.94,0.71)$ & 0.02 \\
\hline \multicolumn{4}{|l|}{ Femoral neck BMD, \% } \\
\hline Week 28 & $0.14(-0.70,0.42)$ & $-0.56(-1.38,0.26)$ & NS \\
\hline Week 54 & $-0.26(-0.90,0.39)$ & $-0.30(-1.25,0.64)$ & NS \\
\hline \multicolumn{4}{|l|}{ Trochanter BMD, \% } \\
\hline Week 28 & $0.09(-0.50,0.68)$ & $-0.67(-1.52,0.18)$ & NS \\
\hline Week 54 & $-0.10(-0.75,0.55)$ & $-1.07(-2.02,-0.12)$ & NS \\
\hline \multicolumn{4}{|l|}{ Total hip, BMD, \% } \\
\hline Week 28 & $-0.02(-0.35,0.31)$ & $-0.61(-1.09,-0.12)$ & 0.05 \\
\hline Week 54 & $-0.13(-0.56,0.30)$ & $-0.89(-1.52,-0.27)$ & 0.05 \\
\hline \multicolumn{4}{|l|}{ СТx-1, \% } \\
\hline Week 28 & $-24.6(-33.6,-15.56)$ & $4.2(-8.8,17.14)$ & $<0.01$ \\
\hline Week 54 & $-11.8(-22.2,-1.43)$ & $8.4(-7.11,23.85)$ & 0.03 \\
\hline
\end{tabular}

indicated a significant change from baseline LS BMD in the calcitonin cohort $(1.11 \%, p<0.001)$ but not the placebo cohort. The difference in change in LS BMD between groups was also significant in this sensitivity analysis. Over 28 weeks, the mean percent LS BMD change from baseline in the MITT oral calcitonin cohort was $0.96 \%, p<0.001$; the placebo cohort experienced a nonsignificant LS BMD decrease from baseline. The difference, $0.95 \%$, approached statistical significance $(p=0.069)$.

Among the calcitonin recipients, 50 and 36 individuals were assigned to evening meal or bedtime administration, respectively; the corresponding numbers were 25 and 18 in placebo recipients. Among calcitonin recipients, the LS BMD change did not differ according to the time of administration (dinner $1.25 \%$ vs bedtime $0.87 \%, p=\mathrm{NS}$ ).

Total proximal femur BMD loss was reduced in the oral calcitonin group at both weeks $28(p=0.05)$ and 54 $(p=0.048)$ relative to placebo recipients. The mean percent changes from baseline in femoral neck and trochanter BMD at weeks 28 and 54 were not significant for either cohort nor were there significant differences between the cohorts.

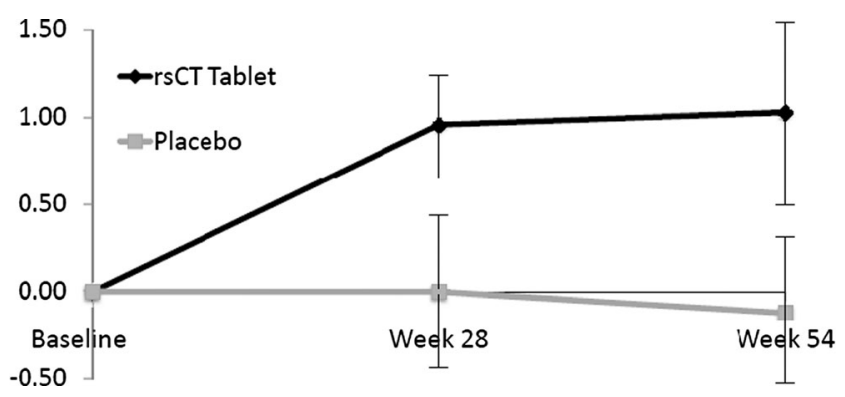

Fig. 2 Mean percent change in lumbar spine BMD over time

\section{Serum CTX-1}

CTx-1 values were significantly reduced from baseline in the calcitonin cohort at weeks 28 and $54(p<0.001$ and 0.041 , respectively) (Table 2 and Fig. 3). The difference between cohorts in CTx-1 values was significant at both time points $(p<0.001$ and 0.034 , respectively). Subjects in the placebo cohort had a numerically higher mean CTx-1 value at both time points; at week 54 , this trended toward significance ( $p$ value $=0.058$ ).

\section{Safety and tolerability}

During the run-in period, 10 women reported 21 AEs and discontinued because of tolerability. The most common system involved was gastrointestinal, with seven $(4.9 \%$ of all patients entering the run-in) events leading to discontinuation. None of these events was classified as severe or serious.

The AEs reported after randomization, i.e., TEAEs, are displayed in Table 3. The proportion of women experiencing any TEAE was comparable between the two groups, $81.4 \%$

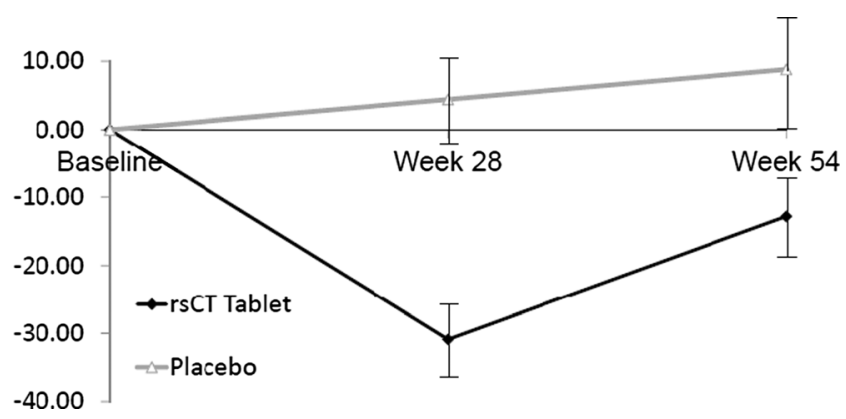

Fig. 3 Mean percent change in CTx-1 over time 
Table 3 Summary of adverse events: safety population

\begin{tabular}{|c|c|c|c|c|}
\hline \multirow[b]{3}{*}{ Adverse event category } & \multicolumn{2}{|c|}{ Oral calcitonin } & \multicolumn{2}{|l|}{ Placebo } \\
\hline & \multicolumn{2}{|l|}{$(N=86)$} & \multicolumn{2}{|l|}{$(N=43)$} \\
\hline & $\mathrm{n}(\%)$ & No. of events & n $(\%)$ & No. of events \\
\hline Any AE & $68(79.1)$ & 211 & $35(81.4)$ & 102 \\
\hline Any SAE & $6(7.0)$ & 9 & $2(4.7)$ & 2 \\
\hline Any treatment-related $\mathrm{AE}$ & $40(46.5)$ & 79 & $25(58.1)$ & 65 \\
\hline Any AE with outcome of death & 0 & $0(0.0)$ & $0(0.0)$ & 0 \\
\hline Any AE leading to discontinuation & $9(10.5)$ & 14 & $5(11.6)$ & 15 \\
\hline \multicolumn{5}{|l|}{ Worst severity } \\
\hline Mild & $24(27.9)$ & & $16(37.2)$ & \\
\hline Moderate & $34(39.5)$ & & $18(41.9)$ & \\
\hline Severe & $10(11.6)$ & & $1(2.3)$ & \\
\hline
\end{tabular}

in the placebo cohort and $79.1 \%$ in the oral calcitonin group. Severe TEAEs were less common in the placebo cohort (one subject [constipation, $2.3 \%$ ] vs ten subjects [11.6\%], respectively); however, no pattern was obvious in the severe TEAEs of calcitonin recipients: upper abdominal pain, pyrexia, acute hepatitis, sepsis, contusion, fall, laceration, lumbar vertebral fracture, breast cancer, meningioma, and syncope. No clinically meaningful differences in the frequency of moderate TEAEs occurred between the placebo and oral calcitonin cohorts (18 subjects [41.9\%] vs 34 subjects [39.5\%], respectively).

Sixty-five subjects $(50.4 \%)$ experienced a TEAE that was considered by the blinded investigator to be treatment-related. The only treatment-related TEAE experienced by $>10 \%$ of subjects in either cohort was upper abdominal pain in the placebo cohort.
No deaths were reported during the study, and less than $10 \%$ of subjects experienced a serious AE (SAE). Serious TEAEs and TEAEs that led to discontinuation were reported in both cohorts. Two subjects $(4.7 \%)$ in the placebo cohort experienced two SAEs, and six subjects $(7.0 \%)$ in the calcitonin cohort experienced nine SAEs. Neoplasm (one subject had breast cancer in each cohort) and fracture (one fracture each in tibia [screen failure], right hip [not randomized], lumbar vertebrae [calcitonin cohort]) were the only SAEs reported by $>1$ subject, and no SAEs were considered related to the investigational product by the blinded investigator(s).

Five subjects $(11.6 \%)$ in the placebo cohort experienced 15 events that led to discontinuation. Nine subjects $(10.5 \%)$ in the oral rsCT cohort had 14 events that led to discontinuation. The TEAEs leading to discontinuation in the placebo cohort
Table 4 Most commonly reported adverse events: safety population

AEs reported in $\geq 5 \%$ of either study group

\begin{tabular}{|c|c|c|c|c|}
\hline \multirow[b]{3}{*}{ Adverse event category } & \multirow{2}{*}{\multicolumn{2}{|c|}{$\begin{array}{l}\text { Oral calcitonin } \\
(N=86)\end{array}$}} & \multirow{2}{*}{\multicolumn{2}{|c|}{$\frac{\text { Placebo }}{(N=43)}$}} \\
\hline & & & & \\
\hline & $\mathrm{n}(\%)$ & $\begin{array}{l}\text { No. of } \\
\text { events }\end{array}$ & $\mathrm{n}(\%)$ & $\begin{array}{l}\text { No. of } \\
\text { events }\end{array}$ \\
\hline At least one $\mathrm{AE}$ & $68(79.1)$ & 211 & $35(81.4)$ & 102 \\
\hline Upper respiratory tract infection & $8(9.3)$ & 10 & $7(16.3)$ & 8 \\
\hline Abdominal pain upper & $6(7.0)$ & 9 & $6(14.0)$ & 9 \\
\hline Arthralgia & $10(11.6)$ & 11 & $1(2.3)$ & 1 \\
\hline Nausea & $8(9.3)$ & 11 & $3(7.0)$ & 5 \\
\hline Abdominal discomfort & $9(10.5)$ & 11 & $1(2.3)$ & 1 \\
\hline Constipation & $6(7.0)$ & 6 & $3(7.0)$ & 4 \\
\hline Abdominal pain & $5(5.8)$ & 6 & $2(4.7)$ & 2 \\
\hline Diarrhea & $5(5.8)$ & 5 & $2(4.7)$ & 5 \\
\hline Nasopharyngitis & $4(4.7)$ & 4 & $3(7.0)$ & 3 \\
\hline Urinary tract infection & $5(5.8)$ & 7 & $1(2.3)$ & 1 \\
\hline Pain in extremity & $2(2.3)$ & 3 & $3(7.0)$ & 3 \\
\hline
\end{tabular}


were upper abdominal pain (three subjects [7\%]), and one subject each with abdominal pain diarrhea, abdominal discomfort, dry mouth, oral lichen planus, and decreased appetite. The TEAEs leading to discontinuation in the oral calcitonin cohort were upper abdominal pain (three subjects [3.5\%]) and abdominal pain (one subject [1.2\%]), and one subject each with diarrhea, nausea, acute hepatitis sepsis, and meningioma.

The most commonly reported AEs, i.e., those occurring in $\geq 5 \%$ of either study arm, are shown in Table 4 . The pattern of AEs was similar across groups, with gastrointestinal AES being reported most commonly; however, arthralgia was reported more often in calcitonin recipients.

\section{Discussion}

In this phase 2 study, oral calcitonin increased LS BMD, reduced total proximal femur BMD loss, and reduced serum CTx1 in women with low bone mass and increased risk of fracture. These results are similar to those observed in an earlier study using this formulation of oral calcitonin compared to placebo and nasal spray calcitonin in women with osteoporosis by BMD [13]. The populations enrolled in these two studies were demographically similar, although the women in the present study had higher baseline BMD based upon the study design.

Oral calcitonin was generally well tolerated. The safety findings did not materially differ by treatment assignment, and few women in either group experienced serious AEs. Gastrointestinal AEs were common in both groups but were typically mild and resolved upon discontinuation.

As calcitonin is a less potent antiresorptive than bisphosphonates or the RANK-L inhibitor denosumab, it is not surprising that the LS BMD improvement among oral calcitonin recipients is modest compared to those agents. However, it is likely that the antiresorptive potency of those agents accounts for some of the $\mathrm{AE}$ association with these medications, e.g., ONJ and AFF. To our knowledge, the complications associated with profound suppression of bone remodeling such as $\mathrm{ONJ}$ and $\mathrm{AFF}$ have never been reported with calcitonin.

Osteoporosis treatment is declining, even following hip fracture $[7,8]$. The reasons behind this are doubtlessly multifactorial; however, concerns about long-term safety are likely in part responsible for this decline. Indeed, some physicians and patients are reluctant to initiate bisphosphonate therapy in view of concerns about AFF, even though the overall risk/benefit profile is favorable. Based on concern about rare side effects, a drug holiday has been recommended after 3-5 years of bisphosphonate use for low-risk patients, even though there is a lack of prospective clinical evidence supporting this recommendation, and the optimal duration of both holiday and long-term use is unknown $[15,17,18]$. In this context, less potent antiresorptive agents such as calcitonin may be more acceptable to some patients and physicians.

However, concerns regarding AEs with calcitonin have also been raised. Recently, the US prescribing information for injectable and nasal calcitonins was revised to reflect a "possible association between malignancy and calcitoninsalmon use," although no (black) boxed warning was added, and no mechanism has been identified [12]. This putative association has been discussed at great length and is of uncertain biological plausibility. For example, individuals with medullary thyroid carcinoma, which may elaborate high levels of human calcitonin, are not at increased risk of second malignancy [19]. Moreover, no signal of carcinogenicity was apparent in formal preclinical animal carcinogenicity testing or in spontaneous AEs reported to the FDA [20]. We have previously reported that the frequency of AEs consistent with malignancy was not elevated in this trial, in the larger phase 3 trial, or when data for these two trials were integrated [21, 22].

Limitations of our study include an absence of fracture data; the revised prescribing information for nasal spray calcitonin notes "fracture reduction efficacy has not been demonstrated." Calcitonin did not improve hip BMD, but our study may have been underpowered to detect such differences.

In summary, based on modest effects on bone resorption and BMD, oral calcitonin may provide a useful alternative for postmenopausal women for whom other therapeutic classes are contraindicated or poorly tolerated.

Acknowledgments The authors thank Richard Trout Ph.D. for statistical support.

Conflicts of interest DK and JG are full time employees of Tarsa Therapeutics, Inc., a company engaged in the development of oral calcitonin, and hold stock in Tarsa Therapeutics, Inc. NB and HB received research funding from Tarsa Therapeutics, Inc. All authors had full access to all raw data, statistical analyses, and material used in the study.

Funding Funding was provided by Tarsa Therapeutics, Inc.

Ethical approval This study was approved by the appropriate ethical standard and institutional review board(s) and was conducted in accordance with the ethical standards laid forth in the 1964 Declaration of Helsinki and its later amendments. Written informed consent was obtained from all participants.

Open Access This article is distributed under the terms of the Creative Commons Attribution Noncommercial License which permits any noncommercial use, distribution, and reproduction in any medium, provided the original author(s) and the source are credited.

\section{References}

1. Rosen CJ (2001) Treatment of postmenopausal osteoporosis: an evidence-based approach. Rev Endocr Metab Disord 2:35-43

2. Rosen CJ (2005) Clinical practice. Postmenopausal osteoporosis. N Engl J Med 353:595-603 
3. Siris ES, Miller PD, Barrett-Connor E et al (2001) Identification and fracture outcomes of undiagnosed low bone mineral density in postmenopausal women: results from the National Osteoporosis Risk Assessment. JAMA 286:2815-2822

4. Kanis JA (2010) Development and use of FRAX in osteoporosis. Osteoporos Int 21(Suppl2):S407-S413

5. Siris ES, Boonen S, Mitchell PJ et al (2012) What's in a name? What constitutes the clinical diagnosis of osteoporosis? Osteoporos Int 23: 2093-2097

6. Siris ES, Adler R, Bilezikian J et al (2014) The clinical diagnosis of osteoporosis: a position statement from the National Bone Health Alliance Working Group. Osteoporos Int 25:14391443

7. Wysowski DK, Greene P (2013) Trends in osteoporosis treatment with oral and intravenous bisphosphonates in the United States, 2002-2012. Bone 57:423-428

8. Solomon DH, Johnston SS, Boytsov NN, et al (2014) Osteoporosis medication use after hip fracture in U.S. patients between 2002 and 2011. J Bone Miner Res Feb 18 (epub)

9. Chesnut CH 3rd, Azria M, Silverman S et al (2008) Salmon calcitonin: a review of current and future therapeutic indications. Osteoporos Int 19:479-491

10. Kittur SD, Hoppener JW, Antonarakis SE et al (1985) Linkage map of the short arm of human chromosome 11: location of the genes for catalase, calcitonin, and insulin-like growth factor II. Proc Natl Acad Sci U S A 82:5064-5067

11. Nuesch E, Schmidt R (1980) Comparative pharmacokinetics of calitonins. In: Pecile A (ed) Calcitonin 1980. Proc Int Symp Milan; pp 352-364

12. Miacalcin ${ }^{\circledR}$ (calcitonin-salmon) nasal spray package insert (2014) Novartis Pharmaceuticals Corporation, East Hanover, NJ
13. Binkley N, Bolognese M, Sidorowicz-Bialynicka A et al (2012) A phase 3 trial of the efficacy and safety of oral recombinant calcitonin: the Oral Calcitonin in Postmenopausal Osteoporosis (ORACAL) trial. J Bone Miner Res 27:1821-1829

14. Qvist P, Christgau S, Pederesen BJ et al (2002) Circadian variation in the serum cncentration of C-terminal telopeptide of Type I collagen (Serum CTx): effects of gender, age, menopausal stautus, posture, daylight, serum cortisol, and fasting. Bone 31:57-61

15. NOF (2014) Clinician's guide to prevention and treatment of osteoporosis. National Osteoporosis Foundation, Washington

16. U.S. Preventative Services Task Force (2011) Screening for osteoporosis: U.S. Preventative Services Task Force recommnedation statement. Ann Int Med 154:356-364

17. Black DM, Bauer DC, Schwartz AV et al (2012) Continuing bisphosphonate treatment for osteoporosis - for whom and for how long? N Engl J Med 366:2051-2053

18. Whitaker M, Guo J, Kehoe T et al (2012) Bisphosphonates for osteoporosis-where do we go from here? N Engl J Med 366: 2048-2051

19. Ronckers CM, McCarron P, Ron E (2005) Thyroid cancer and multiple primary tumors in the SEER cancer registries. Int J Cancer 117:281-288

20. Food and Drug Administration (2013) Background document for meeting of Advisory Committee for reproductive health drugs and drug safety and risk management advisory committee

21. Krause D, Hernandez NAS, Vitagliano M et al (2012) One year use of oral recombinant salmon calcitonin is not associated with increased risk of cancer. J Bone Miner Res 27(suppl 1)

22. Wells G, Krause D, Chernoff J et al (2013) Does calcitonin-salmon cause cancer? Poster session presented at American Society of Bone and Mineral Research Annual Meeting, Oct 4-7, Baltimore MD 